Revista de Derecho

\title{
La regulación de las monedas digitales: experiencias compartidas desde el derecho europeo y francés
}

\author{
The Regulation of Digital Currencies: Shared \\ Experiences from the European and French Law
}

\section{Luis Roman Arciniega Gil}

Investigador en Univ. Lille, CNRS, UMR 8026 - CERAPS - Centre d'Études et de Recherches Administratives Politiques et Sociales, F-59000

Lille, Francia

luisroman.arcniegagil.etu@univ-lille.fr

ORCID: 0000-0001-7162-8952

Artículo de investigación

DOI: https://doi.org/10.32719/26312484.2021.36.2

Fecha de recepción: 28 de diciembre de 2020

Fecha de revisión: 19 de enero de 2021

Fecha de aceptación: 16 de marzo de 2021

Fecha de publicación: 1 de julio de 2021 


\section{RESUMEN}

El presente artículo aborda el fenómeno social de creación monetaria, en el mundo digital, que ha ido creciendo de manera constante en los últimos años. El objetivo es compartir la experiencia del derecho europeo y francés en su intento por determinar el estado jurídico de las monedas digitales, particularmente a la luz de la Ilamada "cadena de bloques" como el aspecto técnico que caracteriza su creación y funcionamiento. La metodología empleada se basa en investigación documental y análisis de las diversas fuentes jurídicas, concretamente de la doctrina, las leyes y la jurisprudencia. El estudio apunta a que es primordial una regulación de la cadena de bloques como el elemento base de estas; un enfoque pragmático en la calificación jurídica de tal elemento técnico conforme a los diversos usos de los que puede ser objeto; así como la necesidad de un acuerdo internacional armonizado que considere su carácter transfronterizo en vista de brindar seguridad jurídica a los usuarios. Como conclusión se tiene que las monedas digitales no pueden ser calificadas como monedas de curso legal. No obstante, el hecho de que sean socialmente aceptadas como medios de pago, así como su carácter multifacético que las presta a diversos fines, hace que los actores del derecho opten por una posición pragmática encuadrándolas jurídicamente conforme al uso que de ellas se hace.

PALABRAS CLAVE: creación monetaria, democratización monetaria, cadena de bloques, monedas digitales, criptomonedas, activos digitales, monedas virtuales, regulación digital.

\section{ABSTRACT}

This article addresses the social phenomenon of monetary creation, in the digital world, that has been growing steadily in recent years. The aim is to share the experience of the European and French law in its attempt to determine the legal status of the so-called digital currencies, particularly considering the blockchain as the technical aspect that characterizes their creation and operation. The methodology employed is based on documentary research and analysis of the various legal sources, specifically doctrine, legislation, and case law. The study points out that a regulation of the blockchain as the basic element of cryptocurrencies is essential; a pragmatic approach in the legal qualification of such technical element according to the different uses that can be made of it; as well as the need for a harmonized international agreement considering its cross-border nature to provide legal certainty to users. In conclusion, digital currencies cannot be qualified as legal tender. However, the fact that they are socially accepted as means of payment, as well as their multifaceted character that lends them to various purposes, make the legal actors to adopt a pragmatic approach framing them legally according to the use that is made of them.

KEYWORDS: monetary creation, monetary democratization, blockchain, digital currencies, crypto-currencies, digital assets, virtual currencies, digital regulation. 


\section{INTRODUCCIÓN}

L a moneda forma parte de un conjunto de cosas que han sido creadas por el intelecto humano, colectivo, siendo objeto de estudio de varias ciencias como la economía, el derecho, la antropología, la sociología y la política, entre otros. Percibida en principio como un medio para satisfacer las necesidades y el deseo de intercambio entre los hombres, la moneda se distingue por tener distintas formas según la cultura y la época. Por ejemplo, primero en forma de trueque, luego con formas más rústicas como el instrumento monetario, finalmente con el dinero en efectivo, letras de cambio, crédito, tarjetas, etc. ${ }^{1}$ Con la llegada de la revolución digital, la moneda evoluciona nuevamente tomando la forma de monedas virtuales, criptomonedas, tokens, etc., y su circulación transfronteriza, favorecida por el internet, transforma las relaciones humanas dando lugar a una dinámica socioeconómica que desafía los marcos jurídicos monetarios vigentes. ${ }^{2}$

No existiendo una definición legal de la moneda, el derecho se ha apoyado en la doctrina económica para considerar como medio de pago aquel instrumento que cumple tres funciones económicas simultáneas. Es decir, la moneda debe servir como (1) intermediario de cambio, (2) unidad de cuenta y (3) reserva de valor.

Así pues, desde la época de la antigua Grecia, Aristóteles, dentro de su Ética a Nicómaco, ya hacía hincapié en las funciones de unidad de cuenta y reserva de valor como elementos característicos de la moneda. ${ }^{3}$ Por otro lado, en su obra La política, también destaca el papel de la moneda como instrumento de intercambio. ${ }^{4}$ En efecto, se considera que los bienes se miden con un criterio, la necesidad recíproca, que es el vínculo de la comunidad de intereses. ${ }^{5}$ En consecuencia, el sustituto de tal necesidad es la moneda o el numisma que existe "no por naturaleza, sino en virtud de la ley (nomos)"; como "medida", la moneda "hace que las cosas sean conmensurables entre sí y así las lleva a la igualdad"; en la ausencia temporal de intercambio, la moneda también es una "especie de prenda" que, aunque sujeta a las mismas fluctuaciones que los demás bienes, se caracteriza por tener una "mayor estabilidad". ${ }^{6}$ Aristóteles ofrece

1. Philipe Rospabé, La dette de vie: Aux origines de la monnaie, coll. Bibliothèque de M.A.U.S.S. (París: Ed. La Découverte, 1995).

2. European Central Bank, Virtual currency schemes, 13-9 (Frankfurt am Main), https://bit.ly/2Xz6sCw.

3. Aristóteles, Ética nicomáquea, trad. Jules Tricot (al francés) (París: J. Vrin, 1990), 240-4.

4. Aristóteles, La política, trad. Jules Tricot (al francés) I, 9 (París: J. Vrin, 1962), 57-8.

5. Jean-Pierre Potier, "D'Aristote à Thomas d'Aquin: Les grands thèmes", Ressources en Sciences économiques et sociales (27 de julio de 2007), http://bit.ly/38D7Qu1.

6. Aristóteles, Ética nicomáquea. 
además una célebre explicación de la transición del trueque a la moneda (intercambio), afirmando que

porque las diversas cosas necesarias para nuestras necesidades naturales no siempre son fáciles de transportar, se convino en dar y recibir a cambio un material que, útil por sí mismo, se manejaba fácilmente en los usos habituales de la vida; se trataba del hierro, por ejemplo, la plata, o alguna otra sustancia similar, cuyo tamaño y peso se determinó en primer lugar, y que, finalmente, para liberarse del bochorno de las continuas mediciones, se marcó con una impronta particular, signo de su valor. ${ }^{7}$

En efecto, la moneda ha existido en varias épocas de la historia, dentro de diferentes civilizaciones y en distintos territorios. A manera de ejemplo, el antiguo Egipto dinástico se caracterizaba por la coexistencia de diversos tipos de moneda y la Europa occidental medieval también tenía varias monedas en circulación al mismo tiempo. ${ }^{8}$ $\mathrm{Al}$ otro lado del Atlántico, la Mesoamérica prehispánica se caracterizó también por utilizar el cacao como moneda de cambio. ${ }^{9}$ No obstante, si bien la moneda es originalmente fruto de una necesidad de intercambios entre los hombres, con el tiempo, la creación monetaria se ha institucionalizado, cumpliendo una función social, servir de intermediaria, pero cuya creación ha sido delegada al Estado como parte de sus facultades soberanas, concretamente mediante el reconocimiento de autoridades monetarias..$^{10}$ En ese sentido, el sistema monetario se define actualmente por dos características principales: (1) una creación de la moneda legal basada en deuda, cuya emisión mediante la concesión de prestamos de los bancos comerciales representa el $90 \%$ de la oferta monetaria; (2) un monopolio concedido a los bancos centrales sobre la emisión legal de divisas, en el que también tienen el rol de supervisores del sistema. ${ }^{11}$

Varios de los proyectos monetarios recientes, por ejemplo, bitcóin, nacen, empero, de la crítica a este monopolio del sector bancario y financiero. En particular, se cuestiona el modelo de creación monetaria basado en deuda, ${ }^{12}$ así como el rol de este sector

7. Aristóteles, La política.

8. Bernard Lietaer et al., Money and Sustainability: The Missing Link. A report from the Club of Rome-EU Chapter to Finance Watch and the World Business Academy (Triarchy Press, 2012), 131-2.

9. Louise Paradis, "Le cacao précolombien: monnaie d'échange et breuvage des dieux", Journal d'agriculture traditionnelle et de botanique appliquée, año 26, Boletín n. ${ }^{\circ}$ 3-4 (julio-diciembre 1979): 181-9. doi:https://doi.org/10.3406/jatba.1979.3800.

10. Christian Lavialle, "La condition juridique de la monnaie fiduciare", Revue française de droit administratif (2009): 669.

11. Lietaer et al., Money and Sustainability: The Missing Link.

12. Philippe Derudder y André-Jaques Holbecq, La dette publique, une affaire rentable. A qui profite le système? (Yves Michel Eds., 2008). 
dentro de las crisis económicas de los últimos años. ${ }^{13}$ Tal circunstancia ha conducido a que ciertos actores reivindiquen un derecho a la creación monetaria, concretamente, mediante la puesta en marcha de nuevas formas de moneda como una manera de hacer frente a los impactos sociales y económicos que dejan las crisis. ${ }^{14}$ En efecto, se tiene evidencia que entre 1970 y 2010, el sistema estuvo sometido a 145 crisis bancarias, 208 crisis monetarias y 72 crisis de deuda soberana, según un análisis de datos del Fondo Monetario Internacional (FMI), presentados por Lietaer et al. $(2012,51)$.

Sin embargo, se tiene evidencia de que la creación monetaria como respuesta de crisis remonta a mucho más tiempo atrás. Para ilustrar, en los años de la Revolución Francesa, las ciudades sitiadas de Lyon y Maguncia introdujeron monedas obsidionales para permitir el comercio intramuros como una respuesta de emergencia local que estaba fuera del control del Estado. ${ }^{15}$ De igual forma, en el primer tercio del siglo pasado, la Gran Depresión de los años 1930 en los Estados Unidos, Alemania, Austria y Suiza, dio lugar a la emisión de una serie de certificados monetarios, varios de ellos creados y gestionados colectivamente. ${ }^{16}$ El WIR suizo, creado en 1934, sigue siendo el proyecto de innovación monetaria más estudiado de aquella época puesto que se trata de un sistema monetario vigente hasta la fecha. ${ }^{17}$ En el mismo sentido, la llamada "crisis del tequila" de 1994 dio lugar a la emisión de varias monedas comunitarias entre las que se encuentran el Tlaloc en México y el billete de trueque en la Argentina. ${ }^{18}$

Los anteriores casos, así como más de 50 monedas locales complementarias en Francia y su expansión en otros países europeos, 60 en Alemania y 70 en España y Grecia, ${ }^{19}$ al igual que más de 5000 proyectos contabilizados en todo el mundo hasta 2015, incluyendo las Palmas de Fortaleza en Brasil y las Libras de Bristol en Inglate-

13. David Bollier y Patty Conaty, Democratic money and capital for the commons: strategies for transforming neoliberal finance through commons-based alternatives. A report on a Commons Strategies Group Workshop in cooperation with the Heinrich Böll Foundation, 8-10 de septiembre de 2015, Berlin, 26-28, https://bit.ly/2K8bKS9.

14. Jérôme Blanc, Les monnaies parallèles: Unité et diversité du fait monétaire (París: L'Harmattan, 2001).

15. Christian Lavialle, "La condition juridique de la monnaie fiduciare".

16. Sara Calvo y Andrés Morales Pachon, "Exploring complementary currencies in Europe: a comparative study of local initiatives in Spain and the United Kingdom", Living in Minca Working Papers (septiembre 2014): 5, https://bit.ly/35B2221.

17. Wojtek Kalinowski, "Currency pluralism and economic stability: the Swiss experience" (París: Veblen Institute for Economic Reforms, 2011), 1-4. http://bit.ly/3oEb7Pn; Guillaume Vallet, "A local money to stabilize capitalism: the underestimated case of the WIR", Economy and Society, 45, 3-4 (2016): 479-504, doi:10.1080/03085147.2016.1224146.

18. María Eugenia Santana Echeagaray, "Reinventando el dinero. Experiencias con monedas comunitarias" (tesis doctoral, Centro de Investigaciones y Estudios Superiores en Antropología Social, México).

19. Benedicte Martin, "Le numérique au secours des monnaies locales et complémentaires", Netcom, 32-1/2, 163-82, doi:10.4000/netcom.3000. 
rra, son evidencia de un tipo de democratización del sistema monetario ${ }^{20}$ que reivindica un derecho a la creación monetaria. El surgimiento de las criptomonedas, en particular del bitcóin en 2009, también puede ser considerado un fenómeno de innovación monetaria postcrisis, puesto que el lanzamiento oficial del proyecto tiene sus orígenes después del colapso financiero mundial de $2008 .{ }^{21}$

Desde un punto de vista jurídico, empero, la creación monetaria resulta mayormente compleja. A nivel europeo, esta se encuentra protegida por el instrumento base que da origen a la Unión Europea (UE), mismo que privilegia una política monetaria única a nivel regional. En efecto, el Tratado de Maastricht establece que:

1. El Banco Central Europeo (BCE) tendrá el derecho exclusivo de autorizar la emisión de billetes de banco en la Comunidad (europea). El BCE y los bancos centrales nacionales podrán emitir billetes. Los billetes emitidos por el BCE y los bancos centrales nacionales serán los únicos billetes de curso legal en la Comunidad. 2. Los Estados miembros podrán realizar emisiones de moneda metálica, para las cuales será necesaria la aprobación del $\mathrm{BCE}$ en cuanto al volumen de emisión. ${ }^{22}$

De igual forma, a nivel nacional, y como parte de la adopción en 1992 del referido tratado, la creación monetaria también se encuentra protegida a nivel francés. De facto, como parte del Tratado de Maastricht entre las naciones europeas, Francia cede la parte monetaria de su soberanía en beneficio del BCE, creado para ejercer tal competencia dentro de la zona euro, modificando así los estatutos del Banco de Francia para integrar el Sistema europeo de bancos centrales implementado por el tratado y dirigido por el BCE. ${ }^{23}$ La legislación francesa refuerza entonces el monopolio de creación monetaria mediante su Código Monetario y Financiero (CMF), estableciendo que: "la moneda de Francia es el euro" y "el Banco de Francia es el único organismo autorizado (...) a emitir billetes de curso legal". ${ }^{24}$

En suma, toda creación monetaria distinta del euro carece, en principio, de reconocimiento legal tanto a nivel europeo como francés. No obstante, los hechos muestran

20. Nicolas Meunier, Informe de la misión de estudio "Monedas locales complementarias y sistemas de cambio locales" (en francés), a petición del Ministro de Vivienda, Igualdad de los Territorios y Asuntos Rurales y del Secretario de Estado de Comercio, Artesanía, Consumo y Economía Social y Solidaria de Francia, 8 de abril de 2015, https://bit.ly/3qqHKkb.

21. Ministerios de Economía, de la Acción y de las Cuentas públicas de Francia, "Monnaie et innovations monétaires", 26 de agosto de 2016, párr. 3, http://bit.ly/3q7h6MO.

22. Tratado de la Unión Europea (TUE) / Tratado de Maastricht, art. 105 A.

23. Francia, "Ley 98-357 de 12 de mayo de 1998 por la que se modifica el estatuto del Banco de Francia con miras a su participación en el Sistema Europeo de Bancos Centrales", Diario oficial de la República francesa (DORF), n. ${ }^{\circ} 110$ (13 de mayo de 1998).

24. Francia, Código monetario y financiero, arts. L.111-1 y L141-5. 
que la ausencia de reconocimiento por parte del derecho no impide el surgimiento de facto de nuevas formas de numisma socialmente aceptado. Las llamadas monedas digitales, cuyo carácter transfronterizo desafía incluso el marco jurídico monetario vigente, no son la excepción. ${ }^{25}$ Muestra de ello es el universo de criptomonedas que está creciendo de manera exponencial y planteando como consecuencia desafíos a las autoridades nacionales e internacionales, sobre todo en materia de convertibilidad con impactos palpables dentro de la economía del mundo real. En efecto, de acuerdo con información del sitio CoinMarketCap, el 16 de septiembre de 2018 se contabilizaban 1867 criptomonedas por un valor de 184.000 millones de euros; un año más tarde había 2871 en circulación ( $+54 \%$ en un año) por un valor de 246.000 millones de euros (+34\%); en diciembre de 2020 tales cifras se actualizan para sumar un total de 8105 criptomonedas por un valor superior a 514.000 millones de euros. ${ }^{26} \mathrm{El}$ derecho debe encontrar los medios para encuadrar jurídicamente tal mercado de monedas digitales. La regulación se plantea entonces como la herramienta jurídica a echar mano para enmarcar un fenómeno exponencial de diversificación monetaria en la era digital.

\section{LA COMPLEJIDAD DE LA NATURALEZA JURÍDICA DE LAS MONEDAS DIGITALES}

Desde un punto de vista jurídico, las criptomonedas no pueden ser consideradas como monedas legales puesto que, a diferencia de estas: 1. no dependen de ninguna institución reconocida por el Estado para su emisión; 2. no tienen curso legal en ningún país lo que dificulta la evaluación de su valor y; 3 . no pueden ser ahorradas por lo que tampoco pueden constituir un valor de reserva. Por el contrario, se trata de objetos digitales creados por una comunidad de internautas, también conocidos como "mineros", a base de un algoritmo que genera "fichas" o tokens que luego se asignan a cada "minero" como recompensa por participar en el funcionamiento del sistema. ${ }^{27}$ La tecnología empleada se denomina "cadena de bloques" y se caracteriza por ser un sistema que funciona mediante el almacenamiento y transferencia de información de forma transparente y segura, sin necesidad de que exista un organismo central de control. Además, tales bloques están asegurados con claves privadas que a su vez

25. OCDE, How to deal with Bitcoin and other cryptocurrencies in the System of National Accounts?, Directorate for financial and enterprise affairs statistics and data directorate, 29 de octubre de 2018, COM/SDD/ DAF(2018)1.

26. Plataforma: CoinMarketCap, https://coinmarketcap.com/fr/.

27. Ministerio de Economía y Finanzas de Francia, "Crypto-monnaies, crypto-actifs... Comment s'y retrouver?", 4 de diciembre de 2020, párr. 3-4, http://bit.ly/39opTDw. 
permiten abrir una cartera digital en donde se almacenan unidades criptográficas que luego pueden ser intercambiadas por dinero legal. ${ }^{28}$

En la práctica, aunque nadie está obligado a aceptar las criptomonedas como medio de pago, estas son utilizadas por los individuos para el intercambio de bienes y servicios tanto en el mundo físico como el digital. ${ }^{29}$ Son estos dos últimos puntos los que están planteando desafíos a los poderes públicos puesto que, las criptomonedas, pueden ser convertibles en moneda legal y ser utilizadas dentro de la economía real, lo que plantea dificultades, por ejemplo, en materia de fiscalidad. ${ }^{30}$ Lo anterior ha motivado a que algunos países como Alemania en 2013 o Japón en 2017, reconozcan al bitcóin como un tipo de moneda privada, permitiendo gravar las transacciones efectuadas con este tipo de moneda virtual. ${ }^{31}$

Es imperativo resaltar, además, que las criptomonedas no pueden ser consideradas como dinero electrónico puesto que la reglamentación prevista para tal efecto no permite calificarlas jurídicamente como tal. En efecto, la Directiva 2009/110/CE del Parlamento Europeo y del Consejo, de 16 de septiembre de 2009, sobre el acceso a la actividad de las entidades de dinero electrónico y su ejercicio, así como sobre la supervisión prudencial de dichas entidades; considera como dinero electrónico: "todo valor monetario almacenado por medios electrónicos o magnéticos que representa un crédito sobre el emisor, se emite al recibo de fondos con el propósito de efectuar operaciones de pago [...], y que es aceptado por una persona física o jurídica distinta del emisor de dinero electrónico". ${ }^{32}$ Su transposición en el CMF francés define el dinero electrónico en los mismos términos y establece además que: "[1]as unidades de dinero electrónico se denominan unidades de valor, cada una de las cuales constituye una reclamación incorporada a un valor". ${ }^{33}$ En otras palabras, el dinero electrónico es, en

28. Ministerios de Economía, de la Acción y de las Cuentas públicas de Francia, "Monnaie et innovations monétaires", párr. 5-6.

29. Amaury Perrin, "Le bitcoin et le droit: problématiques de qualification, enjeux de régulation", Gestion \& Finances Publiques, n. ${ }^{\circ}$ 1-2019 (enero-febrero 2019): 84-93, doi: https://doi.org/10.3166/gfp.2019.00014.

30. Ariane Tichit et al., "Les monnaies virtuelles décentralisées sont-elles des dispositifs d'avenir ?", Revue Interventions Économiques, n. ${ }^{\circ} 59$ (2018), párr. 11, doi:10.4000/interventionseconomiques.3771.

31. Ibíd.

32. Directiva 2009/110/CE del Parlamento Europeo y del Consejo, de 16 de septiembre de 2009, sobre el acceso a la actividad de las entidades de dinero electrónico y su ejercicio, así como sobre la supervisión prudencial de dichas entidades, por la que se modifican las Directivas 2005/60/CE y 2006/48/CE y se deroga la Directiva 2000/46/CE (Texto pertinente a efectos del EEE), art. 2, Diario oficial (DO) L267, de 10 de septiembre de 2009.

33. Francia, Código monetario y financiero, art. L315-1; modificado por la Ley $n .^{\circ} 2013-100$ de 28 de enero de 2013 sobre diversas disposiciones de adaptación de la legislación al derecho de la Unión Europea en materia económica y financiera, art. 5, DORF n. ${ }^{\circ} 0024$ de 29 de enero de 2013. 
virtud de la ley (nomos), dinero legal, con la particularidad de que se presenta en una versión desmaterializada.

Las monedas digitales, en cambio, no pueden ser consideradas como dinero de curso legal. Así lo plantea el informe del BCE de 2012 que al respecto prevé que: "una moneda virtual puede definirse como un tipo de moneda digital no regulada, emitida y verificada por sus creadores y aceptada por los miembros de una comunidad virtual concreta". ${ }^{34}$ Las criptomonedas se diferencian entonces del dinero legal en el sentido de que estas son controladas directamente por sus usuarios y tienen como objetivo llevar a cabo transacciones, sin necesidad de una institución intermediaria reconocida por la ley como los bancos u otras instituciones financieras. ${ }^{35}$

En el mismo sentido, la Directiva (UE) 2018/843, de 30 de mayo de 2018, relativa a la prevención de la utilización del sistema financiero para el blanqueo de capitales o la financiación del terrorismo; define la moneda virtual como una

representación digital de valor no emitida ni garantizada por un banco central ni por una autoridad pública, no necesariamente asociada a una moneda establecida legalmente, que no posee el estatuto jurídico de moneda o dinero, pero [que es] aceptada por personas físicas o jurídicas como medio de cambio y que puede transferirse, almacenarse y negociarse por medios electrónicos. ${ }^{36}$

Tal ordenamiento jurídico también consagra la figura de "proveedor de servicios de custodia de monederos electrónicos" como "una entidad que presta servicios para salvaguardia de claves criptográficas privadas en nombre de sus clientes, para la tenencia, el almacenamiento y la transferencia de monedas virtuales". ${ }^{37}$

Como toda directiva europea, su transposición dentro del marco jurídico francés se da mediante la ley n. ${ }^{\circ} 2019-486$, del 22 de mayo de 2019, relativa al crecimiento y a la transformación de las empresas (también conocida como ley PACTE). ${ }^{38}$ Luego entonces, se enmienda el CMF francés retomando la definición establecida por la Di-

34. European Central Bank, Virtual currency schemes, 5.

35. Instituto Nacional del Consumo de Francia (INC), "Les cryptoactifs: qu'est-ce que c'est ?", 24 de septiembre de 2019, párr. 9, http://bit.ly/2XvQWak.

36. Directiva (UE) 2018/843 del Parlamento Europeo y del Consejo, de 30 de mayo de 2018, por la que se modifica la Directiva (UE) 2015/849 relativa a la prevención de la utilización del sistema financiero para el blanqueo de capitales o la financiación del terrorismo, y por la que se modifican las Directivas 2009/138/CE y 2013/36/UE (Texto pertinente a efectos del EEE), art. 1, 2), d, DO L 156 de 19 junio de 2018, 43-74.

37. Directiva (UE) 2018/843 del Parlamento Europeo y del Consejo, de 30 de mayo de 2018, préc., art. 1, 2), d.

38. Francia, Ley $n .^{\circ} 2019-486$ del 22 de mayo de 2019 relativa al crecimiento y a la transformación de las empresas, art. 86, DORF n. ${ }^{\circ} 0119$ de 23 de mayo de 2019. 
rectiva (UE) 2018/843; con la salvedad de que prefiere llamar "activos digitales" a lo que el ordenamiento europeo designa como "monedas virtuales". ${ }^{39}$ Tal diferenciación, mínima pero medular, es destacable puesto que la falta de emisión de las criptomonedas por parte de alguna institución bancaria autorizada, así como la nula garantía de su aceptación como medio de cambio o de pago, ${ }^{40}$ hace que en estricto sentido no pueda acordársele el término de moneda a un objeto que no cumple con los requisitos que el derecho prevé para tal efecto. ${ }^{41}$

En efecto, las monedas legales como el euro y demás divisas o monedas extranjeras están protegidas y garantizadas por ley, y la emisión de valores adversos que tiendan a sustituirlas se encuentra penalmente sancionada. El CMF francés, en concreto, establece que "la moneda de Francia es el euro"42 y el Código Civil que

[e]l pago en Francia de una obligación de pago de una suma de dinero se hace en euros. Sin embargo, el pago puede efectuarse en otra moneda si la obligación así denominada se deriva de una transacción de carácter internacional o de una sentencia extranjera. Las partes podrán acordar [entonces] que el pago se efectúe en una moneda extranjera si el pago se realiza entre profesionales, cuando el uso de una moneda extranjera sea comúnmente aceptado para la transacción en cuestión. ${ }^{43}$

En sentido adverso, el Código Penal dispone que "la puesta en circulación de cualquier signo monetario no autorizado destinado a sustituir a la moneda o a los billetes de banco, que son de curso legal en Francia, se castigará con cinco años de prisión y una multa de 75.000 euros". ${ }^{44}$ Asimismo, "la negativa a recibir monedas o billetes de curso legal en Francia, según el valor por el que sean válidos, se castigará con una multa". ${ }^{45}$

En consecuencia, las monedas virtuales no pueden ser consideradas de iure como monedas de curso legal. No obstante, el hecho de que sean socialmente aceptadas como medios de pago desafía el marco jurídico e institucional vigente. Este hecho se encuentra incluso presente a nivel internacional puesto que la circulación de las

39. Francia, Código monetario y financiero, art. L. 54-10-1.

40. Directiva (UE) 2015/2366 del Parlamento Europeo y del Consejo, de 25 de noviembre de 2015, sobre servicios de pago en el mercado interior y por la que se modifican las Directivas 2002/65/CE, 2009/110/ CE y 2013/36/UE y el Reglamento (UE) n. ${ }^{\circ} 1093 / 2010$ y se deroga la Directiva 2007/64/CE, DO L 337 de 23.12.2015, 35.

41. Amaury Perrin, "Le bitcoin et le droit: problématiques de qualification, enjeux de régulation", art. préc., 84 .

42. Francia, Código monetario y financiero, art. L.111-1.

43. Francia, Código civil, art. 1343-3.

44. Francia, Código penal, art. L442-4.

45. Ibíd., art. R642-3. 
criptomonedas se caracteriza precisamente por tener un carácter transfronterizo. ${ }^{46} \mathrm{En}$ efecto, el enfoque sociológico, y no el institucional, que define la moneda sobre todo como un acuerdo social, es lo que da soporte a este tipo de objetos digitales y su falta de reconocimiento legal, como moneda, no excluye su uso práctico como medio de cambio, de pago, o reserva de valor. ${ }^{47}$ Lo anterior ha conducido a varios autores a definir los diversos tipos de monedas digitales como "monedas contractuales". La característica principal de tal contrato se centra en que la seguridad jurídica otorgada por el Estado, y que justifica el curso legal de la moneda, se cambia por un acuerdo de partes en la que la seguridad es garantizada por una solución tecnológica llamada "la cadena de bloques". ${ }^{48}$

El Tribunal de Justicia de la Unión Europea (TJUE), en su sentencia de 22 de octubre de 2015, n. ${ }^{\circ}$ 264/14, Skatteverket c. David Hedqvist, confirma tal dimensión contractual de las criptomonedas al considerar que: “ ‘bitcóin’ forma parte de las divisas virtuales denominadas 'de flujo bidireccional', que los usuarios pueden comprar y vender con arreglo al tipo de cambio [...], y permiten adquirir bienes y servicios tanto reales como virtuales"; "En el litigio principal, consta que la divisa virtual 'bitcóin' no tiene ninguna finalidad distinta de la de ser un medio de pago y que ciertos operadores la aceptan como tal". ${ }^{49}$

Los poderes públicos, tomando en cuenta su impacto dentro de la economía del mundo real, se ven entonces obligados a considerar una renovación del marco jurídico con el propósito de regular el uso socialmente aceptado de las monedas digitales como medio de pago. A nivel europeo, es la Directiva (UE) 2018/843, de 30 de mayo de 2018, precitada, la que aborda las cuestiones en la materia, particularmente en lo que respecta su uso en el blanqueo de capitales y financiación del terrorismo. En Francia, se trata de la llamada ley PACTE la que aborda el tema en su dimensión de "activos" de valor capitalizable, en concreto mediante la regulación de su emisión y uso entre personas físicas y jurídicas que las aceptan como medios de pago. El carácter multifacético de las monedas digitales, en el sentido de poder ser utilizadas para diversos

46. Luis Roman Arciniega Gil, "A legal analysis of complementary and virtual currencies for Sustainable economic development”, Banking and Financial Services Policy Report, Wolters Kluwer, vol. 39, n. ${ }^{\circ} 7$ (2020): 1-12.

47. Amaury Perrin, "Le bitcoin et le droit: problématiques de qualification, enjeux de régulation", art. préc., 84 .

48. Jérôme Huet, "Le bitcoin, dont la légalité paraît admise, est une sorte de monnaie contractuelle", Revue des contrats, vol. 4, $\mathrm{n} .^{\circ} 113$ (2017): 54-6.

49. Tribunal de Justicia de la Unión Europea, "Sentencia de 22 de octubre de 2015" (Procedimiento prejudicial-Sistema común del impuesto sobre el valor añadido (IVA)-Directiva 2006/112/CE-artículos 2, apartado 1, letra c), y 135, apartado 1, letras d) a f)-Servicios a título oneroso-Operaciones de cambio de la divisa virtual "bitcóin" por divisas tradicionales-Exención), Caso Skatteverket vs. David Hedqvist, de 22 de octubre de 2015 , párr. 12 y 52. 
fines, por ejemplo, como mecanismo de financiamiento, seguro, o simplemente como bienes in commercium, hace, no obstante, que el estudio se extienda a otros ámbitos del derecho más allá de su simple concepción como medio de pago.$^{50}$ Conviene entonces analizar el aspecto técnico de las monedas digitales, es decir, la cadena de bloques, como el elemento base que permite su creación y funcionamiento.

\section{LA REGULACIÓN DE LAS MONEDAS VIRTUALES A PARTIR DE LA CADENA DE BLOQUES: EL CASO DEL SISTEMA FRANCÉS Y EL RECONOCIMIENTO DE LOS "ACTIVOS DIGITALES"}

Tomando en cuenta el carácter técnico de las monedas virtuales, el marco legislativo francés, a diferencia del europeo, se ha enfocado más en regular la cadena de bloques como tecnología, que en reconocer las cripto-monedas como una forma de dinero. En efecto, la Directiva de la UE 2018/843, antes citada, prevé el término de "monedas virtuales", mientras que el marco jurídico francés, por medio de la también antes referida ley PACTE, opta por utilizar la locución "activos digitales", si bien ambas estableciendo la misma definición en ambos casos. Puesto que el derecho también es un terreno para el combate de palabras, resulta imperativo destacar el uso de la palabra "moneda" dentro de la legislación europea, a diferencia de la francesa que la reserva a lo que jurídicamente se reconoce como tal. Es decir, se reserva el uso de la palabra moneda al euro, mismo que se encuentra protegido como "moneda única" conforme lo establecido por el Tratado de funcionamiento de la Unión Europea ${ }^{51}$ y el CMF de Francia. ${ }^{52}$

Así pues, los actores el derecho francés han optado por regular directamente la cadena de bloques como el elemento base que permite la creación y funcionamiento de los llamados "activos digitales". Al respecto, conviene recordar que las criptomonedas son objetos digitales cuyo funcionamiento se basa en redes informáticas que permiten realizar todo tipo de transacciones en el ciberespacio, gracias a una tecnología llamada blockchain o "cadena de bloques" que permite validar tales transacciones entre dos o más individuos. ${ }^{53}$ Esto ha dado origen a una serie de sucesos normativos que ha edificado paulatinamente el marco jurídico de tal elemento técnico dentro del derecho francés.

50. INC, “Les cryptoactifs: qu'est-ce que c'est ?", párr. 38.

51. Tratado de funcionamiento de la Unión Europea, art. 119, 2.

52. Francia, Código monetario y financiero, art. L.111-1.

53. INC, “Les cryptoactifs : qu'est-ce que c'est ?”, párr. 9. 
La Orden n. ${ }^{\circ} 2016-520$ de 28 de abril de 2016 relativa a los bonos de ahorro, es así el primer instrumento a nivel francés que reconoce y define la cadena de bloques como un "dispositivo de registro electrónico compartido". Dicho ordenamiento actualiza el régimen jurídico aplicable a los bonos de ahorro y permite el desarrollo de la intermediación de plataformas de financiación participativa, en particular mediante la creación de una nueva categoría de "minibonos" basados en la cadena de bloques. ${ }^{54}$ Se enmienda entonces el CMF estableciendo que: "[...] la emisión y la transferencia de minibonos podrán registrarse en un dispositivo de registro electrónico compartido que permita la autenticación de estas operaciones [...]". ${ }^{55}$ Además, dado que la cadena de bloques es un objeto digital controlado por humanos, el derecho francés ha optado también por regular a los actores que participan en su creación y funcionamiento. En ese sentido, la Orden n. ${ }^{\circ}$ 2016-1635, de 1 de diciembre de 2016, que refuerza el sistema francés en la lucha contra el blanqueo de capitales y la financiación del terrorismo, es el instrumento que regula inicialmente a los emisores de tales valores sometiéndolos al sistema de lucha contra el lavado de dinero y financiación del terrorismo (ALD/ CFT). ${ }^{56} \mathrm{Tal}$ instrumento es de relevancia puesto que, hasta antes de la promulgación de dicha orden, los sujetos que participaban en la emisión y transferencia de las monedas digitales no estaban sujetos a ningún procedimiento de aprobación, ni a la supervisión de ninguna autoridad reguladora. ${ }^{57} \mathrm{El} \mathrm{CMF}$ define entonces como profesionales del sector a "toda persona que, como profesión habitual, actúa como contraparte o intermediario para adquirir o vender cualquier instrumento que contenga en forma digital unidades de valor no monetario, que puedan ser retenidas o transferidas con el fin de adquirir un bien o un servicio, pero que no representen un crédito del emisor". ${ }^{58}$

Siguiendo ese orden de ideas, dos textos más han sido promulgados con el propósito de encuadrar legalmente el aspecto técnico de la cadena de bloques. Se trata (1) de la Orden $n .^{\circ}$ 2017-1674, de 8 de diciembre de 2017, relativa a la utilización de un dispositivo de registro electrónico compartido para la representación y la transmisión de valores financieros; ${ }^{59}$ así como (2) del Decreto del Consejo de Estado n. ${ }^{\circ}$

54. Francia, Orden $n .^{\circ} 2016-520$, de 28 de abril de 2016, relativa a los bonos de ahorro, art. 2, DORF n. ${ }^{\circ} 0101$ del 29 de abril de 2016.

55. Francia, Código monetario y financiero, art. L. 223-12.

56. Francia, Orden 2016-1635 del 1 de diciembre de 2016 que refuerza el sistema francés de ALD/CFT, art. 2, DORF 0280 del 2 de diciembre de 2016.

57. Informe TRACFIN - Tendencias y análisis de los riesgos de blanqueo de dinero y financiación del terrorismo, 2016, 21.

58. Francia, Código monetario y financiero, L. 561-2 - $7^{\circ} \mathrm{bis}$.

59. Francia, Orden $n .^{\circ}$ 2017-1674, de 8 de diciembre de 2017, relativa a la utilización de un dispositivo de registro electrónico compartido para la representación y la transmisión de valores financieros, DORF $\mathrm{n}$. 0287, de 9 de diciembre de 2017. 
2018-1226, de 24 de diciembre de 2018, relativo a la utilización de un dispositivo de registro electrónico compartido para la representación y transmisión de valores financieros y para la emisión y transferencia de minibonos. ${ }^{60}$ En la especie, el "dispositivo de registro electrónico compartido" a que hacen referencia los citados instrumentos refiere en efecto a la cadena de bloques. Se reconoce así el hecho de que la cadena de bloques pueda ser calificada como un tipo de valor no monetario, que a su vez puede ser transferido como medio de pago.

En su conjunto, los documentos antes citados conforman los cimientos legales de la cadena de bloques en Francia, sin que ello implique un reconocimiento de las monedas digitales como moneda o dinero de curso legal.

El 22 de mayo de 2019, además, se promulga la llamada ley PACTE por medio de la cual se decide consagrar el término de "activos digitales" (excluidos los instrumentos financieros) comprendiendo los tokens emitidos por la Oferta Inicial de Monedas (OIC) y las llamadas monedas virtuales. ${ }^{61}$ Luego entonces, se regula a los profesionales del sector estableciendo la figura de Proveedores de servicios de activos digitales (PSAN) como entes que se encuentran supervisados por la Autoridad de los Mercados Financieros (AMF). La autorización para el funcionamiento de tales proveedores se vuelve obligatoria cuando lleven a cabo la tenencia de activos digitales en nombre de terceros, o realicen acciones de compra/venta de activos digitales contra moneda de curso legal..$^{62}$ La ley PACTE refuerza así el marco jurídico de la cadena de bloques, en su modalidad de "representaciones digitales de valor", que a su vez pueden servir como medios de pago para la compra de bienes y servicios que son cuantificables dentro de la economía del mundo real. ${ }^{63}$

En efecto, el carácter técnico de las monedas virtuales hace que el marco jurídico francés se enfoque más en la regulación misma de la cadena de bloques, sin que el carácter multifacético de las criptomonedas como medios de pago, instrumentos financieros, seguros o bienes, cuestione la regulación de las mondas digitales como dinero de curso legal.

60. Francia, Decreto $n^{\circ}$ 2018-1226, de 24 de diciembre de 2018, relativo a la utilización de un dispositivo de registro electrónico compartido para la representación y transmisión de valores financieros y para la emisión y venta de minibonos, DORF n. ${ }^{\circ} 0298$ del 26 de diciembre de 2018.

61. Francia, Ley $n .^{\circ} 2019-486$ de 22 de mayo de 2019 relativa al crecimiento y a la transformación de las empresas, art. 86, DORF n. ${ }^{\circ} 019$ de 23 de mayo de 2019.

62. Autoridad de los mercados financieros de Francia, "Vers un nouveau régime pour les crypto-actis en France", 15 de abril de 2019, http://bit.ly/2LGKTgD.

63. Luis Roman Arciniega Gil, "A legal analysis of complementary and virtual currencies for Sustainable economic development", 8 . 
La jurisprudencia a nivel europeo, respecto de la naturaleza jurídica de las monedas virtuales, refleja en ese sentido un enfoque pragmático. Es decir, en primer lugar, se define el uso específico que se da a un activo digital en una situación determinada $\mathrm{y}$, en segundo lugar, se determina por analogía el régimen jurídico aplicable vinculado a su uso. ${ }^{64}$ De esta manera, si el activo digital es utilizado como divisa, el tratamiento que debe otorgarse, en principio, es el de divisa. Sin embargo, si de su uso se desprende que el activo digital sirve de instrumento financiero o mercancía, lo pertinente sería entonces acordarle el trato específico correspondiente. El TJUE, en su fallo de 22 de octubre de 2015, n. ${ }^{\circ}$ 264/14, Skatteverket c. David Hedqvist, precitado, considera lo anterior en ese sentido estableciendo que: "Por lo que respecta a su uso en el mundo real, estas divisas virtuales son análogas a las demás divisas intercambiables, y permiten adquirir bienes y servicios tanto reales como virtuales"; "En el litigio principal, consta que la divisa virtual 'bitcóin' no tiene ninguna finalidad distinta de la de ser un medio de pago y que ciertos operadores la aceptan como tal". ${ }^{65}$ En efecto, lo que se juzga aquí es la cadena de bloques como objeto y el uso que de ella se hace como divisa entre personas que la aceptan como tal.

El carácter transfronterizo de las monedas virtuales, empero, apela a que las autoridades monetarias y públicas de los distintos países a nivel internacional armonicen sus criterios, sobre todo en un contexto en que los usuarios pueden ser objeto de distintos tipos de riesgos. ${ }^{66} \mathrm{Al}$ respecto, el Instituto Nacional del Consumidor de Francia advierte sobre: (a) la volatilidad de los precios que puede dar lugar a posibles pérdidas financieras; (b) el riesgo de la pérdida de inversión en la eventualidad de que la cadena de bloques fuese destruida o que un proyecto de OIC resultase infructuoso; (c) la falta de supervisión por parte de una autoridad de control en los diversos países que operan que garantice la legalidad de su uso; (d) la posibilidad de ser víctima de fraude en el caso de OIC; (e) el riesgo de problemas de liquidez de los PSAN; (f) el blanqueo de dinero, la financiación del terrorismo y otras actividades delictivas que podrían verse favorecidas con el carácter anónimo de las transacciones; (g) así como el hecho de que no exista garantía alguna para la recuperación de fondos. ${ }^{67}$ La ley PACTE aborda algunas de las problemáticas anteriores, en concreto, mediante la elaboración de una lista de empresas no fiables identificados por la AMF. Correlativamente, también da a conocer los proyectos que han obtenido la autorización correspondiente para la entre-

64. Amaury Perrin, "Le bitcoin et le droit: problématiques de qualification, enjeux de régulation", art. préc., 84.

65. Tribunal de Justicia de la Unión Europea, "Sentencia de 22 de octubre de 2015", párr. 12 y 52, respectivamente.

66. OCDE, How to deal with Bitcoin and other cryptocurrencies in the System of National Accounts?, párr. 15-17.

67. INC, “Les cryptoactifs: qu'est-ce que c'est ?", párr. 41. 
ga de las OIC. ${ }^{68}$ Dichas soluciones tienen, sin embargo, la limitante de ser implementadas por ahora a escala nacional y no europea.

\section{CONCLUSIÓN}

La creación monetaria es un fenómeno social que se encuentra presente más allá de las consideraciones jurídicas positivas. En ese sentido, la revolución tecnológica obliga a los poderes públicos a regular el uso de las monedas digitales, aun cuando existen temas pendientes como la regulación de su uso financiero, especulación y la falta de consideración de externalidades negativas a nivel internacional, como el lavado de dinero y financiamiento al terrorismo. En tal tesitura, existe la necesidad de una armonización de criterios por parte de los Estados a la luz de la naturaleza transfronteriza y multifacética que caracteriza el funcionamiento de las monedas digitales. Con respecto a este último punto, resulta pertinente considerar el aspecto técnico de la cadena de bloques en el sentido de que lo que cambia son las formas y no el fondo de la prestación de servicios, en concreto mediante el uso de este nuevo medio tecnológico. Por supuesto, ello no debe excluir la posibilidad de abordar más ampliamente el estado jurídico de la creación monetaria desde una óptica diversa, por ejemplo, la sociológica, que considera la moneda ante todo como un acuerdo social.

En efecto, el derecho ya se auxilia de la ciencia económica para reconocer jurídicamente lo que hoy se entiende como moneda de curso legal. No obstante, en ausencia de definición positiva, no existe impedimento para que, con base a lo aportado por otras ciencias, se pueda reconocer legalmente otras formas de moneda. En tales consideraciones, los marcos jurídicos europeo y francés apuntan al reconocimiento indirecto de una diversificación monetaria que es aceptada socialmente. Los instrumentos analizados en este texto, en particular la Directiva (UE) 2018/843 a nivel europeo y la llamada ley PACTE en Francia, reconocen la existencia de "representaciones digitales de valor" distintas de lo que se conoce hoy como dinero legal. Tales actos jurídicos pueden ser vistos como acciones regulatorias por parte del Estado cuyo propósito es el de encuadrar y orientar un mercado emergente de monedas digitales, así como de los actores que en el intervienen. Se brinda entonces seguridad jurídica a los usuarios y supervisa en cierta medida la circulación y uso que de las criptomonedas se hace.

Finalmente, este artículo también pretende resaltar el fenómeno de la creación monetaria como un acto consensuado por parte de los individuos. Futuras líneas de

68. Francia, Ley $n .^{\circ} 2019-486$ de 22 de mayo de 2019 relativa al crecimiento y a la transformación de las empresas, arts. 26 y 26 bis. 
investigación podrían enfocarse en el estudio de la democratización del sistema monetario, así como de la necesaria reconsideración del concepto de moneda, más allá de la visión tradicional que pregona la doctrina económica. En efecto, al igual que el oro y la plata, los billetes de papel, las tarjetas de crédito y el dinero electrónico, la cadena de bloques viene a ser el soporte de una nueva forma de numisma, en el mundo digital, que es aceptada socialmente como medio de pago. La historia muestra que en distintas épocas y diversos territorios ha sido posible la existencia simultanea de diversos tipos de monedas, más allá de su reconocimiento por parte del poder soberano. La proliferación de varias formas de monedas complementarias en diversas partes del mundo, así como de las criptomonedas que circulan sin un territorio específico, no son la excepción. Conviene entonces encaminar el derecho al estudio de la desmonopolización del sistema monetario, garantizando siempre la seguridad jurídica que amerita su diversificación y consolidando un sistema que trabaje de manera interconectada y sostenible.

\section{BIBLIOGRAFÍA}

Arciniega Gil, Luis Roman. "A legal analysis of complementary and virtual currencies for Sustainable economic development". Banking and Financial Services Policy Report, Wolters Kluwer, vol. 39, n. ${ }^{\circ} 7$ (2020): 1-12.

Aristóteles. Ética nicomáquea, traducido por Jules Tricot (al francés). París: J. Vrin, 1990.

—. La política, traducido por Jules Tricot (al francés), I, 9. París, J. Vrin, 1962.

Autoridad de los mercados financieros de Francia. "Vers un nouveau régime pour les cryptoactis en France", 15 de abril de 2019, http://bit.ly/2LGKTgD.

Blanc, Jérôme. Les monnaies parallèles: Unité et diversité du fait monétaire. París: L'Harmattan, 2001.

Bollier, David, y Conaty Patty. Democratic money and capital for the commons: strategies for transforming neoliberal finance through commons-based alternatives. A report on a Commons Strategies Group Workshop in cooperation with the Heinrich Böll Foundation, 8-10 de septiembre de 2015. Berlin, 54 pages. https://bit.ly/2K8bKS9.

Calvo, Sara, y Andrés Morales Pachon. "Exploring complementary currencies in Europe: a comparative study of local initiatives in Spain and the United Kingdom", Living in Minca Working Papers, septiembre de 2014. https://bit.ly/35B2221.

Derudder, Philippe, y Holbecq André-Jaques. La dette publique, une affaire rentable. A qui profite le système? Yves Michel Eds, 2008.

European Central Bank. Virtual currency schemes. Frankfurt am Main, 55 pages. https://bit. ly/2Xz6sCw.

Huet, Jérôme. "Le bitcoin, dont la légalité paraît admise, est une sorte de monnaie contractuelle". Revue des contrats, vol. 4, n. ${ }^{\circ} 113$ (2017): 54-6. 
Informe TRACFIN - Tendencias y análisis de los riesgos de blanqueo de dinero y financiación del terrorismo (en francés), 2016.

Instituto Nacional del Consumo de Francia (INC). "Les cryptoactifs: qu'est-ce que c'est ?", 24 de septiembre de 2019. http://bit.ly/2XvQWak.

Kalinowski Wojtek. "Currency pluralism and economic stability: the Swiss experience", 1-4. París: Veblen Institute for Economic Reforms, 2011. http://bit.ly/3oEb7Pn.

Lietaer, Bernard, Arnsperger Christian, Goerner Sally, y Brunnhuber Stefan. Money and Sustainability: The Missing Link. A report from the Club of Rome-EU Chapter to Finance Watch and the World Business Academy. Triarchy Press, 2012.

Lavialle, Christian. "La condition juridique de la monnaie fiduciare". Revue française de droit administratif (2009): 669-84.

Martin, Benedicte. "Le numérique au secours des monnaies locales et complémentaires". Netcom, 32-1/2: 163-82. doi:10.4000/netcom.3000.

Meunier, Nicolas. Informe de la misión de estudio "Monedas locales complementarias y sistemas de cambio locales” (en francés), a petición del Ministro de Vivienda, Igualdad de los Territorios y Asuntos Rurales y del Secretario de Estado de Comercio, Artesanía, Consumo y Economía Social y Solidaria de Francia, 8 de abril de 2015. https://bit.ly/3qqHKkb.

Ministerio de Economía y Finanzas de Francia. "Crypto-monnaies, crypto-actifs. Comment s'y retrouver ?", 4 de diciembre de 2020. http://bit.ly/39opTDw.

Ministerios de Economía, de la Acción y de las Cuentas públicas de Francia. "Monnaie et innovations monétaires", 26 de agosto de 2016. http://bit.ly/3q7h6MO.

OCDE. How to deal with Bitcoin and other cryptocurrencies in the System of National Accou$n t s$, Directorate for financial and enterprise affairs statistics and data directorate, 29 de octubre de 2018. COM/SDD/DAF(2018)1.

Paradis, Louise. "Le cacao précolombien: monnaie d'échange et breuvage des dieux". Journal d'agriculture traditionnelle et de botanique appliquée, año 26, Boletín n. ${ }^{\circ}$ 3-4 (juliodiciembre 1979): 181-9. doi:https://doi.org/10.3406/jatba.1979.3800.

Perrin, Amaury. "Le bitcoin et le droit: problématiques de qualification, enjeux de régulation". Gestion \& Finances Publiques, n. ${ }^{\circ}$ 1-2019 (enero-febrero 2019): 84-93. doi:10.3166/ gfp.2019.00014.

Plataforma: CoinMarketCap. https://coinmarketcap.com/fr/.

Potier, Jean-Pierre. "D'Aristote à Thomas d'Aquin: Les grands thèmes". Ressources en Sciences économiques et sociales, 27 de julio de 2007. http://bit.ly/38D7Qu1.

Rospabé, Philipe. La dette de vie : Aux origines de la monnaie, coll. Bibliothèque de M.A.U.S.S. París: Ed. La Découverte, 1995.

Santana Echeagaray, María Eugenia. "Reinventando el dinero. Experiencias con monedas comunitarias". Tesis doctoral, Centro de Investigaciones y Estudios Superiores en Antropología Social, México. 
Tichit Ariane, Lafourcade Pascal, y Mazenod Vincent. "Les monnaies virtuelles décentralisées sont-elles des dispositifs d'avenir?". Revue Interventions Économiques, 59 (2018). doi:10.4000/interventionseconomiques.3771.

Vallet, Guillaume. "A local money to stabilize capitalism: the underestimated case of the WIR". Economy and Society, 45: 3-4 (2016): 479-504. doi:10.1080/03085147.2016.122 4146.

\section{Fuentes JURÍDicas}

Francia, Código civil.

-. Código monetario y financiero.

-. Código penal.

- Decreto $n .^{\circ}$ 2018-1226 de 24 de diciembre de 2018, relativo a la utilización de un dispositivo de registro electrónico compartido para la representación y transmisión de valores financieros y para la emisión y venta de minibonos, Diario oficial de la República francesa DORF, n. ${ }^{\circ} 0298$ del 26 de diciembre de 2018.

- Ley 98-357 de 12 de mayo de 1998 por la que se modifica el estatuto del Banco de Francia con miras a su participación en el Sistema Europeo de Bancos Centrales, DORF, n. ${ }^{\circ} 110$, de 13 de mayo de 1998.

- Ley $n^{\circ}$ 2013-100 del 28 de enero de 2013 sobre diversas disposiciones de adaptación de la legislación al derecho de la Unión Europea en materia económica y financiera, $D O R F, \mathrm{n} .{ }^{\circ}$ 0024 de 29 de enero de 2013.

- Ley $n^{\circ} 2019-486$ del 22 de mayo de 2019 relativa al crecimiento y a la transformación de las empresas, $D O R F$, n. ${ }^{\circ} 0119$ de 23 de mayo de 2019.

- Ley $n .^{\circ} 2019-486$ del 22 de mayo de 2019 relativa al crecimiento y a la transformación de las empresas, DORF, n. $^{\circ} 019$ de 23 de mayo de 2019.

-. Orden 2016-1635 del 1 de diciembre de 2016 que refuerza el sistema francés de ALD/CFT, DORF, 0280 del 2 de diciembre de 2016.

- Orden $n .^{\circ} 2016-520$ del 28 de abril de 2016, relativa a los bonos de ahorro, DORF, n. ${ }^{\circ} 0101$ del 29 de abril de 2016.

- Orden $n .^{\circ}$ 2017-1674 del 8 de diciembre de 2017, relativa a la utilización de un dispositivo de registro electrónico compartido para la representación y la transmisión de valores financieros, DORF, n. ${ }^{\circ} 0287$, de 9 de diciembre de 2017.

Tribunal de Justicia de la Unión Europea. "Sentencia de 22 de octubre de 2015" (Procedimiento prejudicial-Sistema común del impuesto sobre el valor añadido (IVA)-Directiva 2006/112/CE-artículos 2, apartado 1, letra c), y 135, apartado 1, letras d) a f)-Servicios a título oneroso-Operaciones de cambio de la divisa virtual "bitcóin" por divisas tradicionales-Exención), Caso Skatteverket vs. David Hedqvist, de 22 de octubre de 2015.

Unión Europea. Tratado de la Unión Europea (TUE) / Tratado de Maastricht. 
-. Tratado de funcionamiento de la Unión Europea.

-. Directiva (UE) 2018/843 del Parlamento Europeo y del Consejo, de 30 de mayo de 2018, por la que se modifica la Directiva (UE) 2015/849 relativa a la prevención de la utilización del sistema financiero para el blanqueo de capitales o la financiación del terrorismo, y por la que se modifican las Directivas 2009/138/CE y 2013/36/UE (texto pertinente a efectos del EEE), Diario oficial (DO) L 156, de 19 junio de 2018.

-. Directiva 2009/110/CE del Parlamento Europeo y del Consejo de 16 de septiembre de 2009, sobre el acceso a la actividad de las entidades de dinero electrónico y su ejercicio, así como sobre la supervisión prudencial de dichas entidades, por la que se modifican las $\mathrm{Di}$ rectivas 2005/60/CE y 2006/48/CE y se deroga la Directiva 2000/46/CE (texto pertinente a efectos del EEE), DO L 267, de 10 de septiembre de 2009.

-. Directiva (UE) 2015/2366 del Parlamento Europeo y del Consejo de 25 de noviembre de 2015, sobre servicios de pago en el mercado interior y por la que se modifican las Directivas 2002/65/CE, 2009/110/CE y 2013/36/UE y el Reglamento (UE) $n .^{\circ} 1093 / 2010$ y se deroga la Directiva 2007/64/CE, DO L 337, de 23 de diciembre de 2015. 